PROCEEDINGS OF THE

AMERICAN MATHEMATICAL SOCIETY

Volume 134, Number 5, Pages 1331-1338

S 0002-9939(05)08380-2

Article electronically published on October 6, 2005

\title{
SPECTRAL RIGIDITY OF GROUP ACTIONS: APPLICATIONS TO THE CASE $\operatorname{gr}\left\langle t, s ; t s=s t^{2}\right\rangle$
}

\author{
OLEG N. AGEEV
}

(Communicated by Michael Handel)

\begin{abstract}
We apply a technique to study the notion of spectral rigidity of group actions to a group $\operatorname{gr}\left\langle t, s ; t s=s t^{2}\right\rangle$. As an application, we prove that there exist rank one weakly mixing transformations conjugate to its square, thereby giving a positive answer to a well-known question.
\end{abstract}

\section{INTRODUCTION}

In [1] we introduced a notion of spectral rigidity of group actions. This notion is interesting only for noncommutative groups (see Remark 8 in 1]). We say that a property holds for a typical element from a topological space $D$ (or a typical element of a topological space $D$ has some property) if the set of elements from $D$ with this property contains a dense $G_{\delta}$ subset of $D$. Following [1, we say that an element $h$ of a group $H$ has spectral rigidity if for a typical $H$-action $T$ the set of essential values of the spectral multiplicity function of $T_{h}$, named $M\left(T_{h}\right)$, is constant. If every element of $H$ has this property, we say that the group $H$ has spectral rigidity. It is easy to see that the notion of spectral rigidity can be considered as one of the invariants with respect to group, or metrical isomorphisms.

It is well known that a typical transformation has rank one, and is weakly mixing. However, it is not conjugate to its square (see [9]). More information about these notations can be found in [5]. The main goal of this paper is to show how the technique to study the spectral rigidity works in the case of actions of a group $G=\operatorname{gr}\left\langle t, s ; t s=s t^{2}\right\rangle$. Let us recall that even in this simple case we have no reach spectral theorem, because $G$ is wild (see [10]), or, in other terms, $G$ is not of type $I$.

\section{Preliminaries And the Basic Result}

Let $T$ be a transformation defined on a non-atomic standard Borel probability space $(X, \mathcal{F}, \mu)$. The set of all transformations (automorphisms of the $\sigma$ - algebra of measurable sets) of $(X, \mu)$ is a Polish (complete metrizable separable) topological

Received by the editors November 20, 2004.

2000 Mathematics Subject Classification. Primary 37A05, 37A15, 37A25, 37A30, 37A35, 28D05, 28D15; Secondary 47A05, 47A35, 47D03.

Key words and phrases. Group actions, ergodic theory, conjugations to its squares.

The author was supported in part by the Max Planck Institute of Mathematics, Bonn, and the Programme of Support of Leading Scientific Schools of the RF (grant no. NSh-457.2003.1). 
group, noted $\operatorname{Aut}(\mu)$, with respect to the weak (or coarse) topology (see [7]) defined by

$$
T_{n} \rightarrow T \Leftrightarrow \mu\left(T_{n}^{-1} A \Delta T^{-1} A\right) \rightarrow 0 \text { for each measurable } A
$$

(we identify transformations if they coincide up to a set of measure zero).

The spectral properties of $T$ are those of the induced unitary operator on $L_{2}(\mu)$ defined by

$$
\widehat{T}: L_{2}(\mu) \rightarrow L_{2}(\mu) ; \quad \widehat{T} f(x)=f(T x) .
$$

This is Koopman's representation of dynamical systems. Therefore, if $T S=S T^{2}$, then $\widehat{S} \widehat{T}=\widehat{T^{2}} \widehat{S}$. Let us recall that a transformation $T$ has rank $n$ if $n$ is the smallest number such that, for any $k$, there exist integers $h_{k, i}$ and towers (columns)

$$
A_{k, i}, T A_{k, i}, \ldots, T^{h_{k, i}-1} A_{k, i}, \quad i=1, \ldots, n,
$$

such that all levels $T^{j} A_{k, i}, 0 \leq j<h_{k, i}, i=1, \ldots, n$, and the remaining set form a measurable partition of $X$, say $\xi_{k}$, and $\xi_{k} \rightarrow \varepsilon$; i.e. for any measurable set $A$ there are $\xi_{k}$-measurable sets $A_{k}$ such that $\mu\left(A \Delta A_{k}\right) \rightarrow 0$ as $k \rightarrow \infty$. A transformation has infinite rank if there is no such number $n$.

In this paper we study metrical properties of some elements of typical $G$-actions. In particular, we answer a well-known question (see [4, [5], 6]) by proving the following theorem:

Theorem 1.1. There exists a weakly mixing rank one transformation conjugate to its square.

For the proof of Theorem 1.1, we apply a group action approach. Given a discrete group $G$, a $G$-action $T$ is any homomorphism $T: G \rightarrow \operatorname{Aut}(\mu)$. The $G$-action $T$ is called free if $\mu\left\{x \in X:(\exists g \neq 1) T_{g} x=x\right\}=0$. The set of all $G$-actions is equipped with the weak topology defined by

$$
T(n) \rightarrow T \Leftrightarrow(\forall g \in G) T_{g}(n) \rightarrow T_{g} .
$$

When $G$ is countable, the weak topology makes the action space, noted $\Omega_{G}$, a Polish space.

Theorem 1.1 is a natural corollary of the following main theorem:

Theorem 1.2. For a typical $G$-action $T, T_{t}$ is a weakly mixing rank one transformation, where $G=g r\left\langle t, s ; t s=s t^{2}\right\rangle$.

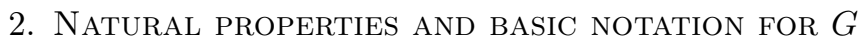

Denote by $Q_{2}$ the subgroup of $G$ generated by all conjugations of $t$, i.e. $g^{-1} t g$, $g \in G$. Following [12], a subset of $G$, say $F$, tiles $G$ if there is a set of centers $C \subseteq G$ such that $\{F c: c \in C\}$ is a partition of $G$.

Proposition 2.1. $\quad$ (1) $Q_{2}$ is an abelian group consisting of elements $s^{-m} t^{n} s^{m}$, $m, n \in \mathbf{Z}$.

(2) $Q_{2}$ is isomorphic to a subgroup of $\mathbf{R}$ via an isomorphism $\phi$ defined by $\phi\left(s^{-m} t^{n} s^{m}\right)=n 2^{m}, m, n \in \mathbf{Z}$.

(3) $G^{\prime}=Q_{2}$.

(4) $G=\bigsqcup_{i \in \mathbf{Z}} s^{i} G^{\prime}$. Therefore $G / G^{\prime} \cong \mathbf{Z}$.

(5) There exists a Følner sequence of sets, say $F_{k}$, such that every $F_{k}$ tiles $G$. 
Proof. The proof is almost obvious. Indeed, part (1) is a consequence of the following equality:

$$
s^{m} t^{p} s^{-m} s^{q} t^{r} s^{-q}=s^{q} t^{r+p 2^{q-m}} s^{-q}=s^{q} t^{r} s^{-q} s^{m} t^{p} s^{-m},
$$

where $q \geq m \geq 0$.

It is easy to see that the map $\phi$ is well defined. Using (2.1) once more, we prove part (2). The statement

$$
\left(\forall g \in Q_{2}\right)\left(\exists g_{1}, g_{2} \in Q_{2}\right)\left[g s^{ \pm 1}=s^{ \pm 1} g_{1} \& g t^{ \pm 1}=g_{2}\right]
$$

is trivial. This implies that $G=\bigsqcup_{i \in \mathbf{Z}} s^{i} Q_{2}$, because $s^{i} \in Q_{2}$ iff $i=0$.

It is easy to check that $\left[g_{1}, g_{2}\right] \in Q_{2}$ for $g_{j}=s^{i_{j}} q_{j}, q_{j} \in Q_{2}$. Thus $G^{\prime} \subseteq Q_{2}$.

To prove both parts (3) and (4), it remains to mention that $Q_{2} \subseteq G^{\prime}$, because $t=\left[s^{-1} t, s\right] \in G^{\prime}$.

Part (5), where we replaced $G$ by $Q_{2}$, holds for some sequence of subsets, say $F_{k}^{\prime}$, of $Q_{2}$. Therefore $\bigcup_{0<i<k} s^{i} F_{k}^{\prime}$ is the sequence we need in part (5). Proposition 2.1 is proved.

\section{Approximations}

3.1. The density of conjugations to any fixed free action in $\Omega_{G}$. For any countable group $G$, the set of free actions is dense in $\Omega_{G}$ (see 3]). Thus we only need to approximate a free action of $G$, say $T^{\prime}$, by conjugations of a fixed free action $T$ of $G$. Given a finite set $F \subseteq G$, and a measurable set $A \subseteq X$ with $\mu(A)>0$, $F \times A$ is said to form a (Rokhlin) tower if the sets $T_{g} A, g \in F$ are mutually disjoint. We say that such a tower is an $(\varepsilon)$-tower if $\mu\left(\bigcup_{g \in F} T_{g} A\right)>\varepsilon$. It said that the Rokhlin lemma is valid for $F$, and a free action $T$ if for all $\varepsilon>0$ there is a measurable set $A$ such that $F \times A$ forms a $(1-\varepsilon)$-tower of $T$. Consider the same sets $F_{k}$ as in Proposition 2.1. The Rokhlin lemma is valid for every $F_{k}$ and every free $G$-action, because $F_{k}$ tiles $G$, and is finite (see [11, and [12 for the proofs of theorems announced in [11]). Therefore we can choose a measurable set $A\left(A^{\prime}\right)$ such that $F_{k} \times A\left(F_{k} \times A^{\prime}\right)$ forms a $(1-1 / k)$-tower for $T$ ( $T^{\prime}$, respectively). Obviously, $A$ and $A^{\prime}$ can be chosen such that $\mu(A)=\mu\left(A^{\prime}\right)$. Therefore there exists a metrical isomorphism $S_{k}: A^{\prime} \rightarrow A$, i.e. a measure-preserving map (onto) which is one-to-one up to zero measure subsets of $A^{\prime}$ and $A$. It is clear that $S_{k}$ can be extended to a metrical isomorphism of $(X, \mu)$ and $(X, \mu)$ (hence it is an automorphism) such that

$$
S_{k} T_{g}^{\prime} x=T_{g} S_{k} x \quad \text { for any } \quad g \in F_{k}, x \in A^{\prime} .
$$

It is easy to see that for any $g \in G$,

$$
S_{k}^{-1} T_{g} S_{k} x=T_{g}^{\prime} x \quad \text { if } x \in T_{g_{1}}^{\prime} A^{\prime} \text { for some } \quad g_{1} \in F_{k} \cap g^{-1} F_{k} .
$$

Therefore,

$$
(\forall g \in G)\left[\mu\left\{x \in X: S_{k}^{-1} T_{g} S_{k} x \neq T_{g}^{\prime} x\right\}<\frac{1}{k}+\frac{\left|F_{k} \backslash g^{-1} F_{k}\right|}{\left|F_{k}\right|} \rightarrow 0 \quad \text { as } k \rightarrow \infty\right] .
$$

This implies that

$$
S_{k}^{-1} T S_{k} \rightarrow T^{\prime} \quad \text { as } k \rightarrow \infty
$$


3.2. An example of a free $G$-action $T$ such that $T_{t}$ is weakly mixing. Let $W$ be a measurable flow on $X$ (i.e. some continuous homomorphism $W$ : $\mathbf{R} \rightarrow \operatorname{Aut}(X, \mu))$ such that the transformation $W^{1}$ is weakly mixing. Define a transformation $T_{0}: \Omega \rightarrow \Omega$ by

$$
T_{0}=\chi_{i=-\infty}^{\infty} W^{2^{i}}
$$

where $\Omega=\prod_{i=-\infty}^{\infty} X$. Also consider the right shift map, say $S$, acting on $\Omega$ by

$$
(S x)_{i}=x_{i-1}, i \in \mathbf{Z},
$$

where $y_{i}$ is the $i$ th coordinate of $y \in \Omega$. Clearly, maps $T_{0}, S$ are weakly mixing transformations of the space $(\Omega, \nu)$, where $\nu=X_{i=-\infty}^{\infty} \mu$, and the map

$$
t \rightarrow T_{0}, \quad s \rightarrow S,
$$

can be naturally extended to a homomorphism from $G$ to a group generated by $T_{0}$ and $S$, so a pair $\left(T_{0}, S\right)$ defines an action of $G$, say $T$. Using Proposition 2.1, to prove the freeness of $T$, we only need to show that

$$
|k|+|l| \neq 0 \Rightarrow \nu\left\{x \in \Omega: T_{0}^{k} x=S^{l} x\right\}=0 .
$$

It is trivial in the case $l=0$, because $W^{1}$ is the aperiodic transformation. To have the same in the case $l \neq 0$, it remains to mention that for any transformation $R$ of a non-atomic standard Borel probability space $(X, \mathcal{F}, \mu)$ we have

$$
\mu \times \mu\{(x, R x): x \in X\}=0 .
$$

This implies that $T$ is a free action.

3.3. An example of a free $G$-action $T$ such that $T_{t}$ has rank 1 . Let $\Lambda$ be a countable discrete subgroup of $\mathbf{T}$ such that the restriction of the map $\phi: \Lambda \rightarrow$ $\Lambda, \phi(\lambda)=\lambda^{2}$, to this subgroup is a group automorphism, and $\Lambda$ has no roots of unity. By the theorem of Pontryagin, the group of characters of $\Lambda$, noted $\widehat{\Lambda}$, is an abelian compact group. It is clear that $\phi^{*}$ is an automorphism of $\widehat{\Lambda}$, where $\phi^{*}$ is defined by $\left(\phi^{*} \chi\right)(\lambda)=\chi(\phi(\lambda))$ for any character $\chi(\lambda)$. Consider a rotation $S: \widehat{\Lambda} \rightarrow \widehat{\Lambda}, S(\chi)=\chi_{0} \chi$, where $\chi_{0}(\lambda)=\lambda$ is a character of $\Lambda$. Maps $\phi^{*}, S$ are transformations of a Lebesgue space $(\widehat{\Lambda}, \mu)$, where $\mu$ is a normalized Haar measure on $\widehat{\Lambda}$. Clearly, $\phi^{*}(\chi)=\chi^{2}$, and $\phi^{*} \circ S=S^{2} \circ \phi^{*}$. All characters of $\widehat{\Lambda}$ form an orthonormal basis of $L_{2}(\widehat{\Lambda}, \mu)$. By the theorem of Pontryagin, every character of $\widehat{\Lambda}$ has the form

$$
\lambda(\chi)=\chi(\lambda)
$$

for some $\lambda \in \Lambda$. Clearly,

$$
\begin{gathered}
\widehat{S} \lambda(\chi)=\lambda(S \chi)=\lambda\left(\chi_{0}\right) \lambda(\chi)=\lambda \lambda(\chi), \\
\widehat{\phi^{*}} \lambda(\chi)=\lambda\left(\chi^{2}\right)=\lambda^{2}(\chi) .
\end{gathered}
$$

Therefore $S$ is an ergodic transformation having discrete spectrum (moreover, $\Lambda_{d}(S)=\Lambda$, where $\Lambda_{d}(S)$ is a set of all eigenvalues of $\widehat{S}$ ). This implies that $S$ has rank 1 (see [8]).

It is clear that a pair $\left(\phi^{*}, S\right)$ defines a $G$-action $T$ via an identification $T_{s}=\phi^{*-1}$, $T_{t}=S$. To prove a freeness of $T$, it is enough to show that

$$
\left(\forall f \in L_{2}\right)(\forall k, l \in \mathbf{Z})\left[\left[{\widehat{\phi^{*}}}^{k} f=\widehat{S}^{l} f\right] \Rightarrow[\text { either } k=l=0 \text { or } f=\text { const }]\right] .
$$


Moreover, we can assume that $k \geq 0$. The transformation $S$ is totally ergodic, because $\Lambda$ has no roots of unity. Therefore (3.1) is true in the case $k=0$. Let $k>0$. Using a uniqueness of a representation of $f$ in the form $\sum_{\lambda} c_{\lambda} \lambda(\chi)$, where $\sum_{\lambda}\left|c_{\lambda}\right|^{2}<\infty$, we have

$$
\left[{\widehat{\phi^{*}}}^{k} f=\widehat{S}^{l} f\right] \Leftrightarrow\left[\sum_{\lambda} c_{\lambda} \lambda^{2^{k}}(\chi)=\sum_{\lambda} c_{\lambda} \lambda^{l} \lambda(\chi)\right]
$$

and $\left|c_{\lambda}\right|=\left|c_{\lambda^{2^{k}}}\right|=\left|c_{\lambda^{2^{k+1}}}\right|=\ldots$

If $\lambda \in \Lambda \backslash\{1\}$, then $\lambda, \lambda^{2^{k}}, \lambda^{2^{k+1}}, \ldots$ are pairwise different. Thus $c_{\lambda}=0$ if $\lambda \neq 1$. Therefore $f \equiv c_{1}$. It follows both (3.1) and the freeness of the $G$-action $T$.

3.4. The density implies the typicality. We need the following modification of Theorem 5 in [1].

Theorem 3.1. Suppose $D$ is a dense subset of $\Omega_{G}$, and $g \in G$; then:

(1) If $T_{g}$ is weakly mixing for any $T \in D$, then $T_{g}$ is weakly mixing for a typical $T$ from $\Omega_{G}$.

(2) If $T_{g}$ has rank 1 for any $T \in D$, then $T_{g}$ has rank 1 for a typical $T$ from $\Omega_{G}$.

Remark 3.2. Let us mention that Theorem 3.1 is also true in the case of any discrete group $G$ such that $\Omega_{G}$ is a Polish space with respect to the weak topology. Moreover, part (2) is true if we replace the property to have rank 1 by the property to have at most rank $k$. The proof of this remark is a certain modification of the proofs of Theorem 3.1 and Theorem 5 in [1].

Proof. Choose a countable subset of $D$, say $\{T(k) \in D: k \in \mathbf{N}\}$, which is dense in $\Omega_{G}$. Let $\varepsilon_{i} \rightarrow 0$ as $i \rightarrow \infty$ for some sequence $\varepsilon_{i}$. Consider

$$
B\left(\varepsilon_{1}, \varepsilon_{2}, \ldots\right)=\bigcap_{k} \bigcup_{i \geq k} U_{\varepsilon_{i}}(T(i)),
$$

where $U_{\varepsilon}(T)=\left\{S \in \Omega_{G}: \rho(T, S)<\varepsilon\right\}, \rho$ is a metric of the space $\Omega_{G}$. It is easy to check that $\Omega_{G}$ has no isolated points. This implies that $\bigcup_{i \geq k} T(i)$ is also dense for any $k$. Therefore $B\left(\varepsilon_{1}, \varepsilon_{2}, \ldots\right)$ is a dense $G_{\delta}$ set.

Obviously, to prove Theorem 3.1, it is enough to find a sequence $\varepsilon_{i}$ such that $B\left(\varepsilon_{1}, \varepsilon_{2}, \ldots\right)$ consists of group actions having properties as required in parts (1) and $(2)$.

Fix a dense sequence of elements in $L_{2}(X, \mu), f_{i}, i=1,2, \ldots$.

To prove part (1), we consider the weak operator topology. Next, we use the well-known fact that a transformation $S$ is weakly mixing if and only if $\widehat{S}^{n_{i}} \rightarrow \int$ for some sequence $n_{i}$, and $\int$ is the operator of orthogonal projection onto the space of constants. Let $0<\delta_{n} \rightarrow 0$ as $n \rightarrow \infty$. Choose a sequence $k_{i}(n)$ such that $\widehat{T}_{g}^{k_{i}(n)}(n) \rightarrow \int$ as $i \rightarrow \infty$. Fix a number $m_{n}=k_{i(n)}(n)$ such that

$$
(\forall p, q \leq n)\left|\left\langle\widehat{T}_{g}^{m_{n}}(n) f_{p}, f_{q}\right\rangle-\left\langle\int f_{p} d \mu, f_{q}\right\rangle\right|<\frac{\delta_{n}}{2} .
$$

Obviously, for any $k \in \mathbf{Z}$ and $h_{1}, h_{2} \in L_{2}(X, \mu)$, the map $\phi: \Omega_{G} \rightarrow \mathbf{R}$ defined by $\phi(T)=\left\langle\widehat{T}_{g}^{k} h_{1}, h_{2}\right\rangle$ is continuous. 
Thus there exists $\varepsilon_{n}>0$ such that

$$
\rho(T(n), T)<\varepsilon_{n} \Rightarrow
$$

$$
(\forall p, q \leq n)\left|\left\langle\widehat{T}_{g}^{m_{n}}(n) f_{p}, f_{q}\right\rangle-\left\langle\widehat{T}_{g}^{m_{n}} f_{p}, f_{q}\right\rangle\right|<\frac{\delta_{n}}{2} .
$$

Let $T \in B\left(\varepsilon_{1}, \varepsilon_{2}, \ldots\right)$, where $\varepsilon_{i}$ were chosen above. Then, for some $n_{j} \rightarrow \infty$, we have $T \in U_{\varepsilon_{n_{j}}}\left(T\left(n_{j}\right)\right)$. Using (3.2) and (3.3), we obtain that

$$
\left|\left\langle\widehat{T}_{g}^{m_{n_{j}}} f_{p}, f_{q}\right\rangle-\left\langle\int f_{p} d \mu, f_{q}\right\rangle\right|<\delta_{n_{j}} \text { for } p, q=1, \ldots, n_{j} .
$$

Hence

$$
(\forall p, q) \lim _{j}\left\langle\widehat{T}_{g}^{m_{n_{j}}} f_{p}, f_{q}\right\rangle=\left\langle\int f_{p} d \mu, f_{q}\right\rangle
$$

Part (1) is proved.

Let us prove part (2). Let $0<\delta_{n} \rightarrow 0$ as $n \rightarrow \infty$. Since $T_{g}(n)$ has rank 1 , there exist integers $k_{i}(n)$, towers

$$
A_{i}(n), T_{g}(n) A_{i}(n), \ldots, T_{g}^{k_{i}(n)-1}(n) A_{i}(n)
$$

and corresponding partitions $\xi_{i}(n)$ of $X$ such that $\xi_{i}(n) \rightarrow \varepsilon$ as $i \rightarrow \infty$. Choose a sequence $i(n)>n$ such that $\xi_{i(n)}(n) \rightarrow \varepsilon$ as $n \rightarrow \infty$. Obviously, for any $k \in \mathbf{Z}$ the map $\phi: \Omega_{G} \rightarrow \operatorname{Aut}(\mu)$ defined by $\phi(T)=\widehat{T}_{g}^{k}$ is continuous. Thus there exists $\varepsilon_{n}>0$ such that

$$
\begin{gathered}
\rho(T(n), T)<\varepsilon_{n} \Rightarrow \\
\max _{0<j<k_{i(n)}(n)} \mu\left(T_{g}^{j}(n) A_{i(n)}(n) \triangle T_{g}^{j} A_{i(n)}(n)\right)<\frac{\delta_{n}}{k_{i(n)}(n)} \mu\left(A_{i(n)}(n)\right) .
\end{gathered}
$$

Let $T \in B\left(\varepsilon_{1}, \varepsilon_{2}, \ldots\right)$, where $\varepsilon_{i}$ were chosen above. Then, for some $n_{j} \rightarrow \infty$, we have $T \in U_{\varepsilon_{n_{j}}}\left(T\left(n_{j}\right)\right)$.

Consider the following set:

$$
\begin{gathered}
A_{j}^{\prime}=A_{i\left(n_{j}\right)}\left(n_{j}\right) \cap T_{g}^{-1} T_{g}\left(n_{j}\right) A_{i\left(n_{j}\right)}\left(n_{j}\right) \cap \ldots \\
\cap T_{g}^{1-k_{i\left(n_{j}\right)}\left(n_{j}\right)} T_{g}^{k_{i\left(n_{j}\right)}\left(n_{j}\right)-1}\left(n_{j}\right) A_{i\left(n_{j}\right)}\left(n_{j}\right) .
\end{gathered}
$$

Obviously,

$$
T_{g}^{l} A_{j}^{\prime} \subseteq T_{g}^{l}\left(n_{j}\right) A_{i\left(n_{j}\right)}\left(n_{j}\right), \quad l=0, \ldots, k_{i\left(n_{j}\right)}\left(n_{j}\right)-1 .
$$

Therefore the sets $T_{g}^{l} A_{j}^{\prime}\left(l=0, \ldots, k_{i\left(n_{j}\right)}\left(n_{j}\right)-1\right)$ and the remaining part of $X$ form a partition of $X$, say $\eta_{j}$. Using (3.4), we obtain that

$$
\frac{\mu\left(A_{j}^{\prime}\right)}{\mu\left(A_{i\left(n_{j}\right)}\left(n_{j}\right)\right)}>1-\frac{\delta_{n_{j}}}{2} \rightarrow 1 \text { as } j \rightarrow \infty .
$$

This implies that $\eta_{j} \rightarrow \varepsilon$ as $j \rightarrow \infty$, because $\xi_{i\left(n_{j}\right)}\left(n_{j}\right) \rightarrow \varepsilon$ as $j \rightarrow \infty$. Thus $T_{g}$ has rank 1. Theorem 3.1 is proved. 


\section{The Proof of Theorem 1.2}

Proof. To prove Theorem 1.2, it is enough to collect all remarks of Section 3. Namely, denote by $A$ the property of a $G$-action $T$ to have a weakly mixing $T_{t}$. Properties we consider are invariant with respect to any metrical isomorphism (in particular, any conjugation). Therefore the set of actions having the property $A$ is dense. Finally, Theorem 3.1 implies that $A$ holds for a typical $G$-action. It remains to repeat the same arguments for the set of $G$-actions $T$ satisfying the $\operatorname{rank} T_{t}=1$ condition. Theorem 1.2 is proved.

\section{Closing Remarks}

There are many observations about metrical properties of dynamical systems conjugated to their composition squares (see [5], [6]). We restrict our attention to the "typical" case, i.e. we consider metrical properties of $T_{t}$ for a typical $G$-action $T$. It is not difficult to prove that in our case $T_{t}$ is rigid, i.e. $T_{t}^{k_{i}} \rightarrow E$ for some sequence $k_{i}$, where $E$ means the identity transformation. Therefore the typical transformation is not mixing. It is also easy to see that the typical transformation $T_{t}$ has a simple singular spectrum. Moreover, the maximal spectral type of $\widehat{T}_{t}$ is invariant with respect to a map $\phi: \mathbf{T} \rightarrow \mathbf{T}, \phi(\lambda)=\lambda^{2}$.

All statements of this paper can be naturally extended to the cases of both maps $T_{t}^{q}$, where $q=k 2^{l}, k \neq 0, l \in \mathbf{Z}$, and $G_{n}=\operatorname{gr}\left\langle t, s ; t s=s t^{n}\right\rangle[1$ where $n \neq 0$. However let us mention that the case $n=-1$ is essentially different, because (see [1] Theorem 7) for a typical $G_{-1}$-action $T$, transformation $T_{t}$ has a homogeneous spectrum of multiplicity 2 in the orthogonal complement of the constant functions.

\section{REFERENCES}

1. O. Ageev, The homogeneous spectrum problem in ergodic theory, Invent. Math. 160 (2005), 417-446. MR2138072

2. I.P. Cornfel'd , S.V. Fomin, Ya. G. Sinai, Ergodic Theory (Springer-Verlag, 1980). MR0832433 (87f:28019)

3. E. Glasner, J.L. King, A zero-one law for dynamical properties, Topol. Dyn. and Appl. 215 (1998), 231-242. MR.1603201 (99d:28039)

4. G. Goodson, A survey of recent results in the spectral theory of ergodic dynamical systems, J. Dynam. Control Systems 5 (1999), 173-226. MR.1693318 (2000f:28021)

5. G. Goodson, Ergodic dynamical systems conjugate to their composition squares, Acta Math. Univ. Comenian. (N.S.) 71 (2002), 201-210. MR.1980380 (2004c:37008)

6. G. Goodson, Spectral properties of ergodic dynamical systems conjugate to their composition squares, preprint.

7. P.R. Halmos, Lectures on ergodic theory (N.Y.: Chelsea Publ. Comp., 1960). MR0111817 $(22: 2677)$

8. A. del Junco, Transformations with discrete spectrum are stacking transformations, Canad. J. Math. 24 (1976), 836-839. MR0414822 (54:2914)

9. A. del Junco, Disjointness of measure-preserving transformations, self-joinings and category, Prog. Math. K (1981), 81-89. MR0633762 (82m:28035)

10. A.A. Kirillov, Elements of the theory of representations (Springer-Verlag, 1976). MR0412321 $(54: 447)$

\footnotetext{
${ }^{1}$ If $n>1$, then it is a famous solvable Baumslag-Solitar group $B S(1, n)$.
} 
11. D. Ornstein, B. Weiss, Ergodic theory of amenable group actions. I. The Rohlin lemma, Bull. Amer. Math. Soc. (N.S.) 2 (1980), 161-164. MR.0551753 (80j:28031)

12. D. Ornstein, B. Weiss, Entropy and isomorphism theorems for actions of amenable groups, J. Analyse Math. 48 (1987), 1-141. MR0910005 (88j:28014)

Department of Mathematics, Moscow State Technical University, 2nd Baumanscaya St. 5, 105005 Moscow, Russia

E-mail address: ageev@mx.bmstu.ru

Current address: Max Planck Institute of Mathematics, P.O. Box 7280, D-53072 Bonn, Germany

E-mail address: ageev@mpim-bonn.mpg.de 